\title{
RadICAL Stack: A localisation method for dynamic gamma/neutron fields
}

\author{
George Randall, Mark Ellis, Kirk Duroe, Ashley Jones, Malcolm Joyce, Chris Payne, Paul Sellin, Robert Speller
}

\begin{abstract}
A variation of the RadICAL (Radiation Imaging Cylinder Activity Locator) system capable of operating in a dynamic environment, such as that created by active interrogation techniques, has been developed. RadICAL is a novel method for locating a radiological source using a rotating detector element. The detector geometry is that of a thin sheet and is rotated to present a constantly changing surface area to the source; it therefore generates a characteristic temporal response which can be used to determine the source direction. The time required to determine the direction of a source make it unsuitable for dynamic environments and so an alternative method is presented that uses a stack of identical scintillator slabs positioned at fixed horizontal angles around a central axis. By comparing count rates from each slab to a standard response curve, using a specially developed algorithm, the direction of a source can be determined without the need to rotate the detector. EJ-29933 plastic scintillator was used to allow detection of separate neutron and gamma events in a mixed field through pulse shape discrimination. A four element detector was built and shown to achieve a positional accuracy of approximately 4.4 degrees when exposed to a $1.44 \mathrm{MBq}{ }^{137} \mathrm{Cs}$ source at distances of up to $2 \mathrm{~m}$. The same detector was used to discriminate separate neutron and gamma events in a mixed field, which allows for the possibility of locating a neutron source within a gamma rich environment.
\end{abstract}

\section{INTRODUCTION}

$\mathbf{T}$ HIS document describes the development of a directional, neutron/gamma discriminating detection method that was built as part of a collaborative investigation that was undertaken between University College London, the University of Surrey, Lancaster University and AWE to develop a detection system for high flux, dynamic, mixed field active interrogation environments.

\section{A. The RadICAL Concept}

RadICAL (Radiation Imaging Cylinder Activity Locator) uses a detection element whose general shape is that of a thin sheet. When the sheet is presented to the source face-on the area of the detector is at its largest and the radiation pathlength is minimum. When the sheet is turned so that only the edge is presented the area is at its smallest and the pathlength is maximum. Thus if the sheet is continually rotated, as shown in Figure 1, the solid angle subtended by the detector varies as it

G.Randall and R.Speller are with the Department of Medical Physics and Biomedical Engineering, University College London, UK e-mail: george.randall.10@ucl.ac.uk

M.Ellis and K.Duroe are with AWE, Reading, UK

A.Jones and M.Joyce are with the Department of Engineering, University of Lancaster, UK

C.Payne and P.Sellin are with the Department of Physics, University of Surrey, UK.

Manuscript received October 27, 2015; revised November 23, 2015.

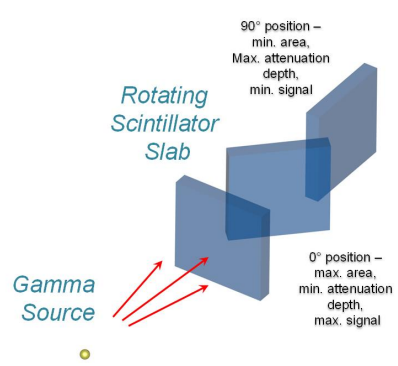

(a) A slab of scintillator rotates to produce the RadICAL response

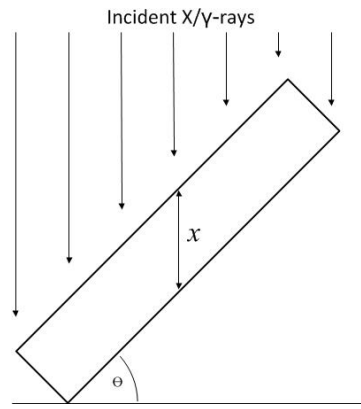

(b) Geometry of scintillator

Fig. 1: The RadICAL concept

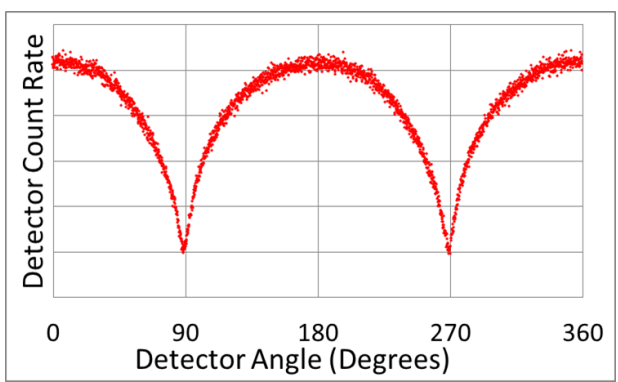

Fig. 2: Standard response of rotating detector exposed to single point source

is rotated in the radiation field and the fluctuating signal forms a characteristic response curve that can be used to locate the direction of a source. The changing pathlength can counteract this effect and alters the overall response shape slightly at higher energies. An example Standard Response Curve (SRC) is shown in Figure 2. The minimum count rates correspond to the angle at which the small face of the scintillator is presented to the source. This response has been tested extensively with a variety of different scintillation detectors, geometries and photon sources and has been demonstrated to be an effective means of locating a radioactive source without the use of a collimator [1]. The fraction of incident photons absorbed by the rotating detector, at each angle, can be expressed as:

$$
\frac{N}{N_{o}}=e^{-\int \mu_{e n}(E) \cdot x(\theta) \cdot d x}
$$

where: $N_{o}=$ Total number of incident photons, $\mathrm{N}=$ number of incident photons that pass through the detector without interacting, $\mu_{e n}(E)=$ attenuation coefficient at energy $E$ and $\mathrm{x}=$ detector thickness at angle $\theta$. 


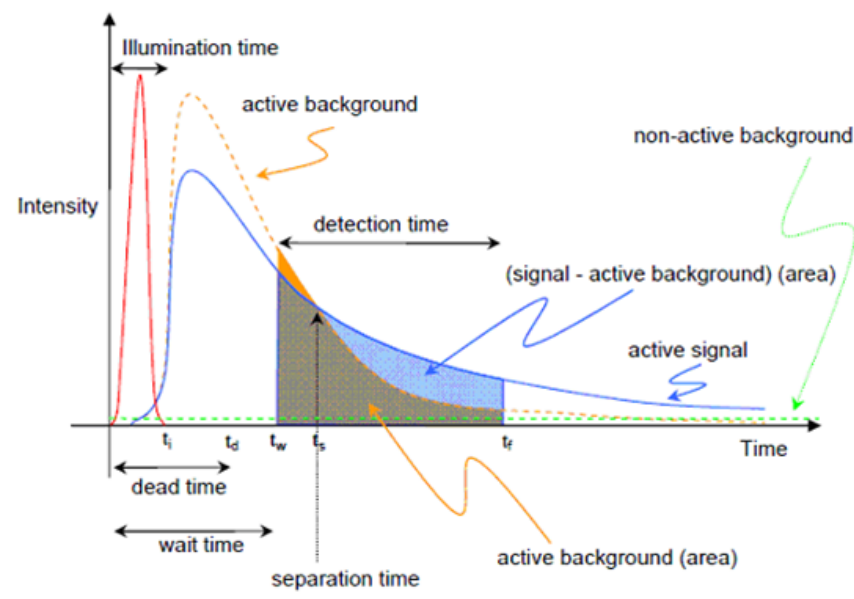

Fig. 3: An example of the dynamic fields observed in active interrogation environments

\section{B. Active Interrogation}

Active interrogation is a method of detecting specific materials of interest by directing nuclear radiation, normally high energy photons or neutrons, at a volume of interest. When bombarded by specific radiation various materials of interest may undergo induced fission that will produce a unique signature in the form of prompt and delayed neutrons and gammas. A common application of this technique is in the detection of special nuclear materials (SNM) such as highly enriched uranium $\left({ }^{235} \mathrm{U}\right)$ [2].

An illustration of an active interrogation environment is shown in Figure 3 and demonstrates the dynamic signal and background intensities that follows an interrogation pulse. The separation time, ts represents the time, relative to the initial interrogation pulse, after which the active signal can be observed above the background.

\section{Neutron/Gamma Identification Using Pulse Shape Discrim- ination}

When using scintillation detectors to detect neutrons it is usually necessary to distinguish them from a larger gamma field. This is normally done by observing the different shaped pulses of electric current from the photodetector which correspond to neutrons or gammas interacting in the scintillator. In most scintillators nearly all of the light produced by scintillation is promptly emitted and can be described by a short decay time component. In some materials, notably Stilbene and various oxygen-free liquid scintillators, a sizeable longer time component is also present. The composite yield curve can often be described adequately by the sum of the two exponential decays, represented by each of these components [3]. The slow component is LET (linear energy transfer) dependent and so becomes pronounced by particles with denser energy deposition tracks such as alpha particles or recoil protons from fast neutron scattering [4].

A new PVT based plastic scintillator, EJ-299-33, has been developed by Eljen to deliver PSD in a similar manner to established liquid scintillators. This offers neutron detection properties without the handling problems associated with liquid organic scintillators. [5].

A recent study, made as part of this collaboration, suggests that the quality of PSD in Eljen EJ-299-33 is shape dependent [6] and this might impose a limit on RadICAL performance.

\section{The RadICAL Stack}

In a dynamic field, such as those investigated during active interrogation, the time required to rotate between positions with a conventional RadICAL detector would invalidate the concept. A proposed variation on the RadICAL concept provides a potential solution to this problem and involves replacing the single rotating detector with a stack of identical scintillators directly coupled to photodetectors and separated at fixed angles around the centre of each slab of scintillator. The source position can be determined by fitting the individual count rates from each of these detectors to a standard response curve using the least squares method.

\section{Methods}

\section{A. Building Four Element Stack Detector}

A 4 element detector stack was built to be sensitive over a $90^{\circ}$ field of view. This involved 4 separate $150 \times 35 \times 12.5 \mathrm{~mm}$ Eljen EJ-299-33 scintillator slabs. The smallest face of each slab was coupled directly to the window of an ET Enterprises 9102B photomultiplier tube (PMT) and the remaining surfaces were then covered in white, diffuse reflective, paint and wrapped in PTFE tape to minimise ambient light penetrating the detector. Each detector was then mounted centrally within a separate, light tight, ABS enclosure. The four detector elements were then stacked vertically and positioned at a $30^{\circ}$ offset to one another around an axis that corresponds with the center of each scintillator slab. A diagram of this setup, including a detailed view of the top detector, is shown in Figure 4. Each PMT was then connected to a HV power supply and a CAEN V1751 (10bit GSample/s) digitiser.

\section{B. Gamma Localisation Procedure}

The detector was initially tested by exposing to a 1.44 $\mathrm{MBq}{ }^{137} \mathrm{Cs}$ source within the UCL laboratory. A set of four Calibration Standard Response Curves ( ${ }_{\mathrm{c}} \mathrm{SRC}$ ) was initially taken by rotating the complete detector stack around $270^{\circ}$ at a distance of $1.55 \mathrm{~m}$ from the source. These ${ }_{\mathrm{c}}$ SRC's were then compared to ensure that an identical shape could be observed for each element. Minor differences were observed in the count rates due to an inconsistency in the efficiency of coupling each element and PMT. This difference proved to be consistent between experiments and so these count rates were normalised to produce a single ${ }_{\mathrm{c}} \mathrm{SRC}$.

The stationary detector stack was then tested with the ${ }^{137} \mathrm{Cs}$ source in 24 different positions, relative to the detector. These positions covered a $60 \times 100 \mathrm{~cm}$ grid as shown in Figure 5 and in all positions the source was kept vertically aligned with the centre of the detector. In each position separate acquisitions were taken in windows of 10,100 and 1000 seconds 


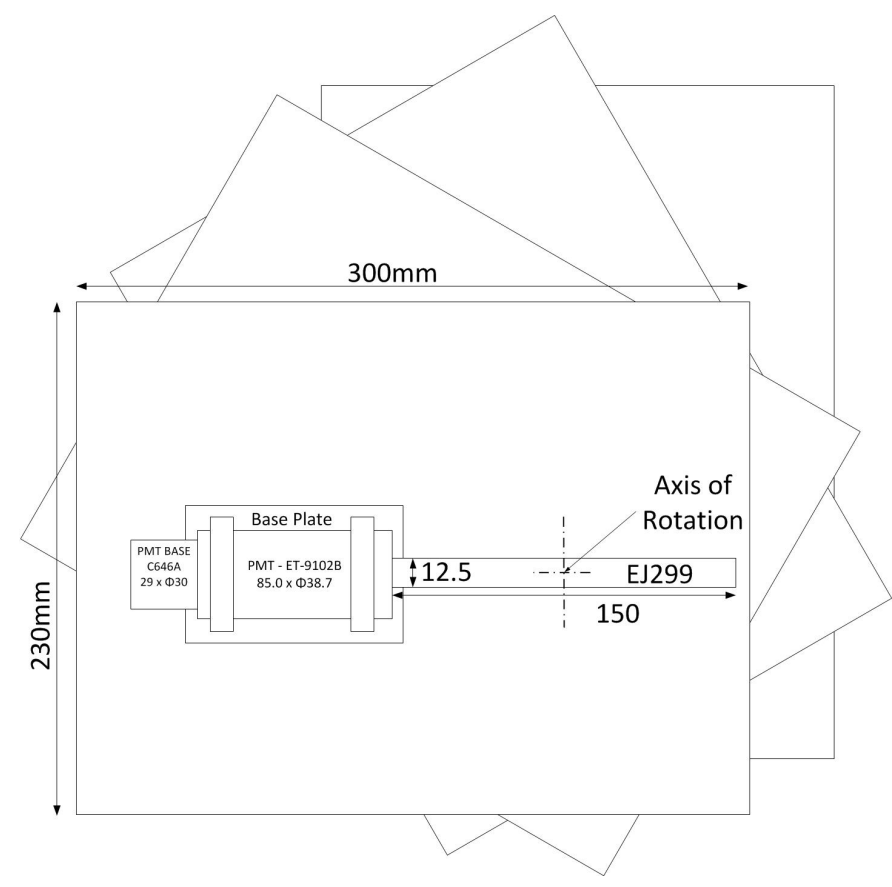

Fig. 4: Plan of 4 element stack detector (all measurements are in $\mathrm{mm}$ )

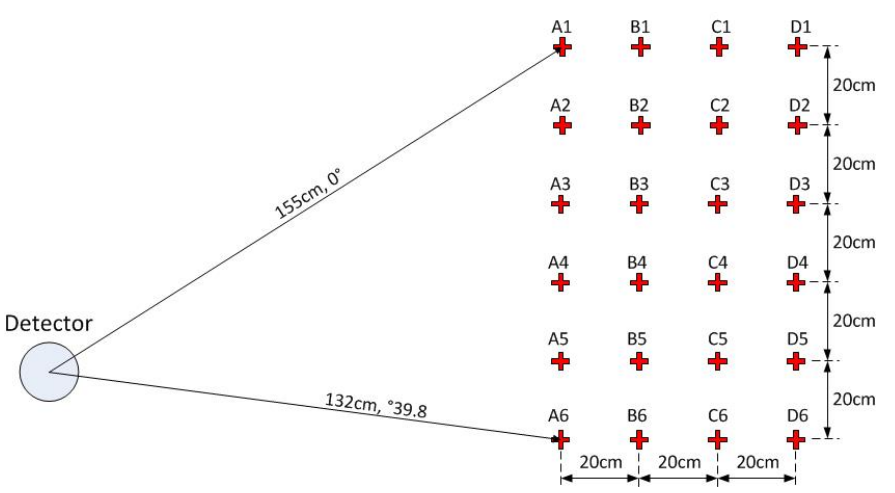

Fig. 5: Position of ${ }^{137} \mathrm{Cs}$ source, relative to stationary detector

For each source position and time window count rates for all four detectors were fit to the ${ }_{\mathrm{c}} \mathrm{SRC}$ using the following method:

- Counts rates were multiplied by a scaling factor that corresponded to the counting efficiency of each detector to allow them to be directly compared.

- These four adjusted count rates were then scaled to fit the count rate of the of the ${ }_{\mathrm{c}} \mathrm{SRC}$. This was done by using the detector with the maximum detected counts to estimate the maximum point on the curve and the max/min ratio of the ${ }_{\mathrm{c}} \mathrm{SRC}$ to estimate the minimum point.

- These scaled detector points were then fit to the ${ }_{c} S R C$ using a least squares method in order to find the direction of the source.

\section{Neutron/Gamma Discrimination}

An investigation was conducted to use the pulse shape discrimination properties of the Eljen EJ-299-33 to identify

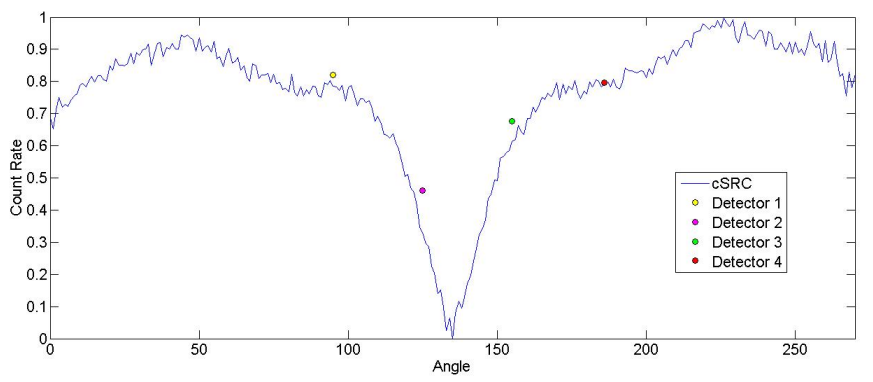

Fig. 6: Normalised standard response of rotating detector and fitted points

separate gamma and neutron events within the detector. This involved exposing the detector to a $150 \mathrm{MBq}{ }^{252} \mathrm{Cf}$ source within a low scatter environment at the National Physical Laboratories in Teddington, UK. The CAEN V1751 was used with its DPP-PSD software [7] to automatically calculate the ratio of two charge integrals that correspond to a long and a short gate for a single event. This value is know as the 'PSD number' and can be plotted against the corresponding energy for each event to discriminate gamma events from neutrons and other heavy particles. The detector was also exposed to a ${ }^{137} \mathrm{Cs}$ to produce an equivalent gamma only plot. Each set of data was then discriminated using the following method:

- The ${ }^{137}$ Cs data was split between 400 different energy bins.

- The events in each of these bins were then placed in a histogram according to their PSD value. For each set this produced an approximately Gaussian shape.

- The PSD value that corresponds to the maximum of each of these distributions and the FWHM were then logged for each energy bin.

- This data was then used to determine a neutron/gamma separation point.

\section{RESULTS}

\section{A. Gamma Localisation Results}

Results for the procedure described in section IIB are shown in Table I. It was shown that the source could be localised to within an average of $4.4^{\circ}$ for 1000 second acquisitions, with the positional accuracy reducing as the acquisition time is reduced. Each detector element registered between approximately 35 and 75 events per second for this set of acquisitions.

\section{B. Neutron/Gamma Discrimination Results}

Figure 7 shows an energy-PSD plot for gamma only events from ${ }^{137} \mathrm{Cs}$. Figure 8 shows an equivalent plot for a mixed gamma and neutron field from ${ }^{252} \mathrm{Cf}$ and depicts two distinct plumes that represent gamma and neutron events. Figure 8 includes a 1 sigma neutron/gamma separation as described in the method detailed in section IIC of this report. Both plots show a distinctive upwards curve in the PSD values as the energy is increased. This is most likely due to high energy pulses exceeding the maximum dynamic input range of the digitiser. A significant overlap between neutron and gamma 


\begin{tabular}{|c|c|c|c|c|c|}
\hline Position & $\begin{array}{c}\text { Measured } \\
\text { Angle (degrees) }\end{array}$ & $\begin{array}{c}\text { Measured } \\
\text { Distance }(\mathrm{mm})\end{array}$ & $10 \mathrm{~s}$ & $100 \mathrm{~s}$ & $1000 \mathrm{~s}$ \\
\hline A1 & 0.0 & 155 & 10.6 & 9.4 & 8.4 \\
\hline A2 & 6.7 & 145.3 & 16.1 & 0.1 & 1.9 \\
A3 & 14.2 & 137.8 & 22.6 & 1.6 & 2.6 \\
A4 & 22.4 & 132.9 & 4.8 & 23.2 & 3.8 \\
A5 & 31.1 & 130.9 & 48.5 & 5.5 & 8.5 \\
A6 & 39.8 & 132 & 0.2 & 1.8 & 2.2 \\
B1 & 4.3 & 174 & 5.3 & 11.7 & 7.7 \\
B2 & 10.3 & 164 & 14.3 & 4.7 & 0.3 \\
B3 & 17.1 & 158 & 0.5 & 0.5 & 6.5 \\
B4 & 24.1 & 153 & 13.5 & 5.5 & 5.5 \\
B5 & 31.9 & 152 & 2.7 & 8.3 & 6.7 \\
B6 & 39.0 & 152 & 17.4 & 0.6 & 2.4 \\
C1 & 6.8 & 191 & 15.8 & 2.8 & 3.8 \\
C2 & 12.6 & 183 & 4.0 & 1.0 & 2.0 \\
C3 & 18.9 & 177 & 3.7 & 7.3 & 2.3 \\
C4 & 25.1 & 173 & 16.5 & 5.5 & 6.5 \\
C5 & 32.0 & 172 & 8.4 & 8.6 & 7.6 \\
C6 & 38.6 & 172 & 2.5 & 1.0 & 0.0 \\
D1 & 9.4 & 210 & 28.2 & 2.2 & 0.2 \\
D2 & 14.0 & 202 & 6.4 & 3.4 & 1.6 \\
D3 & 20.3 & 196 & 0.7 & 22.3 & 2.7 \\
D4 & 26.0 & 193 & 14.6 & 7.4 & 13.6 \\
D5 & 32.3 & 191 & 11.7 & 8.7 & 7.3 \\
D6 & 38.4 & 192 & 16.8 & 3.2 & 1.2 \\
\hline \multicolumn{7}{r|}{ Mean Difference } & 11.9 & 6.1 & 4.4 \\
\hline
\end{tabular}

TABLE I: Gamma localisation results for $1.76 \mathrm{MBq}{ }^{137} \mathrm{Cs}$ source exposed to detector

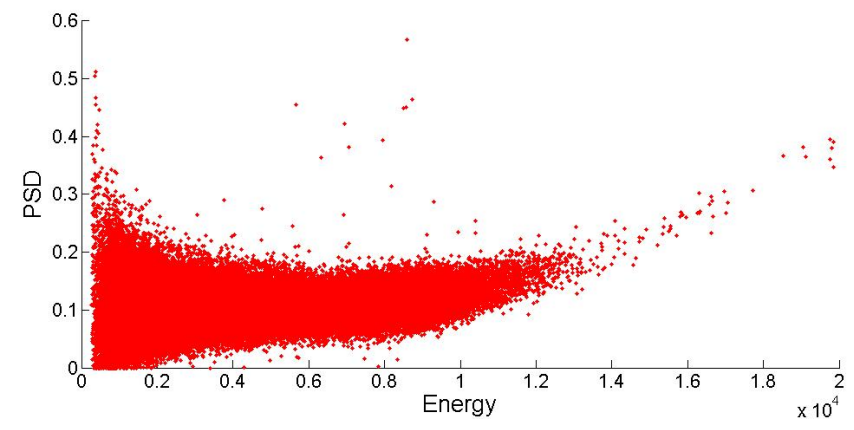

Fig. 7: Energy-PSD plot for ${ }^{137} \mathrm{Cs}$

events is apparent so a higher separation point is used to minimise falsely identified neutrons. By setting a 3 sigma separation for all detector elements just 0.3 percent of ${ }^{137} \mathrm{Cs}$ events were positively identified as neutrons. These represent a mixture of background neutrons and misidentified gammas. For the equivalent ${ }^{252} \mathrm{Cf}$ plot 6 percent of events were identified as neutrons.

\section{CONCLUSION}

The RadICAL Stack concept was demonstrated to be effective and the curve fitting method can be seen to work as an effective alternative to the previously demonstrated RadICAL detection method. Four stationary detector elements can be used to determine directional information about the position of a source Positional accuracy has been demonstrated up to $4.4^{\circ}$ with a greater scope for improvement with higher fluxes. Pulse shape discrimination between neutron and gamma events has been demonstrated using the same detection elements used for imaging which allows the possibility of locating a neutron source within a gamma rich environment. There is scope for

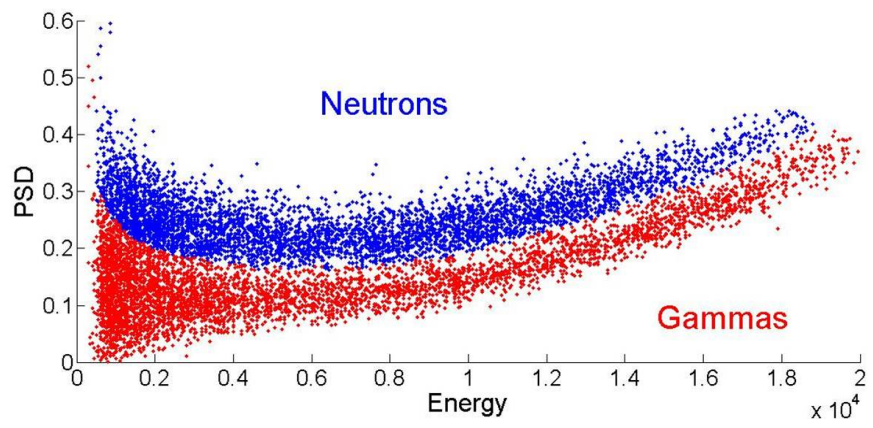

Fig. 8: Energy-PSD plot for ${ }^{252} \mathrm{Cf}$ showing neutron/gamma separation at $1 \mathrm{x}$ sigma

further work to be completed in providing separate neutron and gamma localisations within a mixed field.

\section{REFERENCES}

[1] G. Randall, E. Iglesis, H. F. Wong and R. Speller A method of providing directionality for ionising radiation detectors-RadICAL Journal of Instrumentation, 2014

[2] D. R. Slaughter, M.R. Accatino, A. Bernstein, J.A. Church, M.A. Descalle, T.B. Gosnell, M. McDowell Preliminary results utilizing high-energy fission product gamma-rays to detect fissionable material in cargo Nuclear Instruments and Methods in Physics Research, Section B: Beam Interactions with Materials and Atoms, 2005

[3] G.F. Knoll Radiation Detection and Measurements, 3rd edition John Wiley and Sons Inc., 2000

[4] F.H. Attix Introduction to Radiological Physics and Radiation Dosimetry John Wiley and Sons Inc., 1986

[5] S. A. Pozzi, M.M. Bourne, S.D. Clarke Pulse shape discrimination in the plastic scintillator EJ-299-33 Nuclear Instruments and Methods in Physics Research, Section A: Accelerators, Spectrometers, Detectors and Associated Equipment, 2013

[6] C. Payne, P.J. Sellin, M. Ellis, K. Duroe, A. Jones, M. Joyce, G. Randall, R. Speller Neutron/gamma pulse shape discrimination in EJ-299 at high flux IEEE Nuclear Sciences Symposium, 2015

[7] CAEN Digital Pulse Processing for Pulse Shape Discrimination User Manual UM2580, 2015

(C) British Crown Owned Copyright 2015/AWE 